\title{
Drug Use History
}

National Cancer Institute

\section{Source}

National Cancer Institute. Drug Use History. NCI Thesaurus. Code C81246.

A description of an individual's current and past use and/or abuse of drugs and chemicals.

This includes prescription medication, illicit drugs and recreational substances. 